\title{
Seleção simultânea de clones de eucalipto de acordo com produtividade, estabilidade e adaptabilidade
}

\author{
Antônio Marcos Rosado(1), Tatiana Barbosa Rosado(2), Alexandre Alonso Alves ${ }^{(2)}$, \\ Bruno Galvêas Laviola(2) e Leonardo Lopes Bhering ${ }^{(3)}$
}

\begin{abstract}
${ }^{(1)}$ Celulose Nipo-Brasileira, Rodovia BR 381, Km 172, CEP 35196-000 Belo Oriente, MG. E-mail: antonio.rosado@yahoo.com.br (2)Embrapa Agroenergia, Parque Estação Biológica, s/no, CEP 70770-901 Brasília, DF. E-mail: tatianarosado@yahoo.com.br, alexandre.alonso@embrapa.br, bruno.laviola@embrapa.br ${ }^{(3)}$ Universidade Federal de Viçosa, Avenida P.H. Rolfs, s/no, Campus Universitário, CEP 36570-000 Viçosa, MG. E-mail: leonardo.bhering@embrapa.br
\end{abstract}

\begin{abstract}
Resumo - O objetivo deste trabalho foi estimar os parâmetros genéticos e determinar a eficiência da seleção simultânea de clones de eucalipto baseada em produtividade, estabilidade e adaptabilidade. Foram utilizados 21 clones, com 36 meses de idade, pertencentes ao programa de melhoramento genético da empresa Cenibra. O experimento foi instalado em delineamento de blocos ao acaso, em quatro ambientes, com 21 repetições de uma planta por parcela. Os clones foram avaliados quanto às variáveis: diâmetro à altura do peito, altura da planta e volume total com casca. Os parâmetros genéticos foram estimados pela metodologia de modelos mistos (REML/BLUP), e a seleção baseou-se no método da média harmônica do desempenho relativo dos valores genéticos (MHPRVG), em três estratégias: seleção com base no valor genético predito, tendo-se considerado o desempenho médio dos genótipos em todos os ambientes (sem efeito de interação) ou o desempenho em cada ambiente (com efeito da interação); e seleção simultânea quanto à produção, estabilidade e adaptabilidade. As particularidades dos ambientes influenciaram a expressão fenotípica dos clones. As estimativas de herdabilidade indicaram boas perspectivas para a seleção de clones com alta produtividade, estabilidade e adaptabilidade. A seleção simultânea otimiza a seleção de clones e pode ser usada na construção de populações de melhoramento e na recomendação de materiais genéticos para plantios comerciais.
\end{abstract}

Termos para indexação: Eucalyptus, interação genótipo x ambiente, MHPRVG, REML/BLUP.

\section{Simultaneous selection of eucalyptus clones based on yield, stability and adaptability}

\begin{abstract}
The objective of this work was to estimate genetic parameters and to determine the efficiency of the simultaneous selection of eucalyptus clones based on yield, stability, and adaptability. Twenty-one clones, with 36 months of age, from the genetic breeding program of the company Cenibra were used. The experiment was done in a randomized complete block design, in four environments, with 21 replicates of one plant per plot. The clones were evaluated as to the variables: diameter at breast height, plant height, and total volume with bark. The genetic parameters were estimated using the mixed model procedure (REML/BLUP), and selection was based on the harmonic mean of the relative performance of the genetic values (HMRPGV), with three strategies: selection based on the predicted genetic gain, considering the average performance of genotypes in all environments (without interaction effect) or the performance on each environment (with interaction effect); and simultaneous selection based on productivity, stability, and adaptability. Environment particularities influenced the phenotypic expression of the clones. The heritability estimates indicated good perspectives for selecting clones with high yield, stability, and adaptability. Simultaneous selection optimizes clone selection and can be used in the construction of breeding populations and in the recommendation of genetic materials for commercial plantations.
\end{abstract}

Index terms: Eucalyptus, genotype x environment interaction, HMRPGV, REML/BLUP.

\section{Introdução}

A área de plantios clonais de Eucalyptus vem sendo ampliada cada vez mais em todo o território brasileiro, principalmente em decorrência das vantagens do processo de multiplicação e da possibilidade de se contornar problemas de heterogeneidade nos plantios florestais. Além disso, com a clonagem, têm-se observado consideráveis avanços nos programas de melhoramento que buscam o aumento da produtividade de madeira (Fonseca et al., 2010).

Nos programas de melhoramento, um grande número de clones promissores é testado anualmente em diferentes ambientes, antes de sua recomendação final e multiplicação (Sudaric et al., 2005). Uma vez que, na maioria das vezes, estes ambientes são distintos, há interação entre genótipo e ambiente (GxE), o que

Pesq. agropec. bras., Brasília, v.47, n.7, p.964-971, jul. 2012 
afeta o ganho com a seleção e torna necessário estimar a magnitude e a natureza dessa interação. Essas estimativas possibilitam a avaliação do real impacto de seleção e asseguram alto grau de confiabilidade na recomendação de clones para um determinado local ou grupo de ambientes.

No entanto, apesar de sua importância, a simples análise da interação GxE não proporciona informações completas e exatas sobre o comportamento de cada genótipo em várias condições ambientais. Para tanto, devem ser realizadas análises de adaptabilidade e estabilidade fenotípica, pelas quais é possível a identificação de genótipos com comportamento previsível, que sejam responsivos às variações ambientais, em condições específicas ou amplas (Cruz et al., 2004). Nesse sentido, metodologias de seleção que incorporam a estabilidade e a adaptabilidade em uma única estatística podem ser consideradas superiores, em comparação àquelas que usam apenas a produção como critério de seleção (Resende, 2007b; Verardi et al., 2009).

O método da média harmônica do desempenho relativo dos valores genéticos (MHPRVG), proposto por Resende (2007a), baseia-se na análise dos valores genéticos por meio de modelos mistos e permite efetuar a seleção de genótipos por meio da consideração simultânea de produtividade, estabilidade e adaptabilidade dos clones. O modelo considera os efeitos genotípicos como aleatórios e, portanto, fornece a estabilidade e a adaptabilidade genotípica, o que permite a análise de dados desbalanceados e de delineamentos não ortogonais e com heterogeneidade de variâncias. Além disso, o modelo permite considerar erros correlacionados dentro de locais, bem como a estabilidade e a adaptabilidade na seleção de indivíduos dentro de progênies; fornece valores genéticos já descontados da instabilidade; e pode ser aplicado a qualquer número de ambientes. Ademais, ele gera resultados na própria unidade ou escala do caráter avaliado, que podem ser interpretados diretamente como valores genéticos, o que os outros métodos não permitem. Esse modelo também permite inferências adicionais, tais como: seleção de genótipos específicos para cada local; seleção de genótipos estáveis através dos locais; seleção de genótipos responsivos (com alta adaptabilidade) à melhoria do ambiente; e seleção pelos três atributos (produtividade, estabilidade e adaptabilidade), simultaneamente.
As estatísticas média harmônica dos valores genéticos (MHVG), desempenho relativo dos valores genéticos (PRVG) e média harmônica do desempenho relativo dos valores genéticos (MHPRVG) preditos têm sido usadas como medidas para interpretação da estabilidade genotípica e da adaptabilidade de plantas perenes, e são empregadas como distintos critérios à simulação de seleção por produtividade, estabilidade e adaptabilidade. Em estudo realizado com eucalipto, Pinto Junior et al. (2006) verificaram que as estatísticas MHVG, PRVG e MHPRVG mostraram-se úteis à simulação da seleção de indivíduos, ao fornecer opções para a escolha de estratégias e critérios de seleção mais oportunos, com base nos distintos valores de ganhos genéticos preditos. Para outras espécies, a metodologia também tem sido usada com sucesso e contribui para aumentar a eficiência nos programas de melhoramento com vistas à seleção e à recomendação de material genético para cultivo em diferentes condições edafoclimáticas (Sturion \& Resende, 2005; Zeni-Neto et al., 2008; Bastos et al., 2009; Maia et al., 2009; Verardi et al., 2009).

O objetivo deste trabalho foi estimar os parâmetros genéticos e determinar a eficiência da seleção simultânea de clones de eucalipto baseada em produtividade, estabilidade e adaptabilidade.

\section{Material e Métodos}

Foram utilizados 21 clones de eucalipto pertencentes ao programa de melhoramento da empresa Celulose Nipo-Brasileira S.A. (Cenibra), localizada no Município de Sabinópolis, MG, em teste clonal implantado em dezembro de 2001. O clone 386, que faz parte dos clones comerciais, já recomendados para plantio, foi usado como testemunha. O experimento foi instalado em quatro ambientes (Tabela 1), em delineamento em blocos ao acaso, com 21 repetições e uma planta por parcela, no espaçamento de $3 \times 2 \mathrm{~m}$. As práticas silviculturais foram realizadas de acordo com as particularidades exigidas por cada ambiente, conforme manual de recomendações técnicas para cultivo de eucalipto da Cenibra.

Os clones foram avaliados, aos 36 meses, quanto ao diâmetro à altura do peito (DAP, $\mathrm{cm}$ ), à altura total (ALT, $\mathrm{m}$ ) e ao volume individual total com casca (VOL, $\mathrm{m}^{3}$ ). $\mathrm{O}$ volume individual total com casca foi calculado por meio da expressão: $\mathrm{VOL}=0,004761+0,000033 \times \mathrm{DAP}^{2} \times \mathrm{ALT}^{2}$. 
As estimativas dos parâmetros genéticos foram obtidas com base no procedimento Reml/Blup, com uso do modelo estatístico 54 do Selegen (Resende, 2007b), dado por $\mathrm{y}=\mathrm{Xr}+\mathrm{Zg}+\mathrm{Wi}+\mathrm{e}$, em que: $\mathrm{y}$ é vetor de dados de efeitos fixos (médias de blocos através dos locais), efeitos genotípicos de clones (aleatório), efeitos da interação genótipo $\mathrm{x}$ ambiente (aleatório) e de erros ou resíduos (aleatórios); e X, $\mathrm{Z}$ e W são as matrizes de incidência para $\mathrm{r}$, $\mathrm{g}$ e $\mathrm{i}$, respectivamente.

Para estudo da interação genótipo $\mathrm{x}$ ambiente, foi usado o modelo parcimonioso misto univariado, de efeitos principais $(\mathrm{G})$ e interação (GxE), que reproduz, aproximadamente, via $\mathrm{G}+\mathrm{GE}$, os resultados do modelo Blup multivariado (Resende, 2007a). O Blup dos efeitos GxE elimina os chamados ruídos da interação genótipo $\mathrm{x}$ ambiente. Isto pode ser verificado ao se considerar a predição Blup obtida a partir de Tabela de dupla entrada, com genótipos ( $\mathrm{g}$ ) e ambiente (e) contendo as médias de cada genótipo, em cada ambiente. $\mathrm{O}$ modelo associado a esta Tabela é o seguinte: $\mathrm{Y}_{\mathrm{ij}}=\mu+\mathrm{g}_{\mathrm{i}}+\mathrm{e}_{\mathrm{j}}+\mathrm{ge}_{\mathrm{ij}}+\mathrm{e}_{\mathrm{ij}}=\overline{\mathrm{Y}}+\left(\overline{\mathrm{Y}}_{\mathrm{i}}-\overline{\mathrm{Y}}\right)+\left(\overline{\mathrm{Y}}_{\mathrm{j}}-\overline{\mathrm{Y}}\right)$ $+\left(\bar{Y}_{i j}-\bar{Y}_{i}-\bar{Y}_{j}+\bar{Y}\right)+e_{i j} ;$ em que e $e_{i j}$ é o resíduo associado às médias, em cada ambiente. A predição BLUP da média genotípica, em cada local $\left(\mu+\mathrm{g}_{\mathrm{i}}+\mathrm{e}_{\mathrm{j}}+\mathrm{ge}_{\mathrm{ij}}\right)$, é dada por: $\mathrm{I}=\overline{\mathrm{Y}}+\mathrm{h}_{\mathrm{g}}^{2}\left(\overline{\mathrm{Y}}_{\mathrm{i}}-\overline{\mathrm{Y}}\right)+\mathrm{h}_{\mathrm{e}}^{2}\left(\overline{\mathrm{Y}}_{\mathrm{ij}}-\overline{\mathrm{Y}}_{\mathrm{i}}-\overline{\mathrm{Y}}_{\mathrm{j}}+\overline{\mathrm{Y}}\right)$, quando se considera os efeitos de ambiente como aleatórios. Os ponderadores dos índices são: herdabilidade dos efeitos de genótipos,

$$
\mathrm{h}_{\mathrm{g}}^{2}=\left(\mathrm{S}_{\mathrm{g}}^{2}+\mathrm{S}_{\mathrm{ge}}^{2} / \mathrm{L}\right) /\left(\mathrm{S}_{\mathrm{g}}^{2}+\mathrm{S}_{\mathrm{g}}^{2} / \mathrm{L}+\mathrm{S}_{\mathrm{e}}^{2} / \mathrm{L}_{2}\right) ;
$$

herdabilidade dos efeitos da interação GxE,

$$
\mathrm{h}_{\mathrm{ge}}^{2}=\mathrm{S}_{\mathrm{ge}}^{2} /\left(\mathrm{S}_{\mathrm{g}}^{2}+\mathrm{S}_{\mathrm{e}}^{2}\right)
$$

e coeficiente de determinação dos efeitos de ambiente,

$$
\mathrm{h}_{\mathrm{e}}^{2}=\left(\mathrm{S}_{\mathrm{e}}^{2}+\mathrm{S}_{\mathrm{ge}}^{2} / \mathrm{L}\right) /\left(\mathrm{S}_{\mathrm{e}}^{2}+\mathrm{S}_{\mathrm{ge}}^{2} / \mathrm{G}+\mathrm{S}_{\mathrm{e}}^{2} / \mathrm{G}\right) ;
$$

em que $\mathrm{G}$ e $\mathrm{L}$ referem-se aos números de genótipos e locais, respectivamente, e $S$ à variância residual associada às médias. O BLUP GxE, a partir desses índices, considera a herdabilidade dos efeitos da interação GxE, ou seja, elimina os ruídos ou os efeitos residuais por ocasião do processo de predição da interação.

A seleção dos melhores clones foi baseada no método MHPRVG e consistiu de três estratégias: seleção baseada no valor genético predito, tendose considerado o desempenho médio em todos os ambientes (sem efeito de interação); seleção baseada no valor genético predito, tendo-se considerado o desempenho dos genótipos em cada ambiente (com efeito da interação média); e seleção simultânea quanto à produção, à estabilidade (MHVG) e à adaptabilidade (PRVG). Todas as análises foram realizadas com auxílio do programa Selegen (Resende, 2007b).

\section{Resultados e Discussão}

As estimativas das variâncias genotípicas $(\mathrm{Vg})$ mostraram que os três caracteres avaliados nos ambientes em estudo apresentaram taxas de controle genético similares, guardadas as devidas escalas (Tabela 2). Como essas estimativas foram elevadas, fica evidente a existência de variação genética entre os clones, o que permite inferir que há boas chances de sucesso na seleção quanto aos três caracteres. As estimativas dos parâmetros genéticos são importantes no direcionamento dos programas de melhoramento, uma vez que auxiliam o processo seletivo e servem como referencial teórico para suporte às recomendações dos materiais comerciais (Maia et al., 2009).

A herdabilidade é um dos mais importantes parâmetros genéticos, pois quantifica a fração da variação fenotípica de natureza herdável, passível de ser explorada na seleção. A herdabilidade da média de genótipos $\left(\mathrm{h}^{2}{ }_{\mathrm{mg}}\right)$ é estimada quando se usam médias como unidade de avaliação/seleção. Neste caso, atinge-se nível significativo de precisão em razão da diminuição dos erros experimentais pelo incremento proporcional do número de repetições.

\begin{tabular}{|c|c|c|c|c|c|}
\hline Ambientes & Região & Topografia & Temperatura média anual $\left({ }^{\circ} \mathrm{C}\right)$ & Índice pluviométrico $(\mathrm{mm})$ & Tipo de solo \\
\hline 1 & Virginópolis, MG & Encosta & 19,0 & 1.131 & Cambissolo \\
\hline 2 & Santa Bárbara, MG & Encosta & 21,7 & 1.472 & Latossolo \\
\hline 3 & Belo Oriente, MG & Baixada & 24,4 & 1.243 & Latossolo \\
\hline 4 & Cocais, $\mathrm{MG}$ & Encosta & 20,0 & 1.285 & Cambissolo \\
\hline
\end{tabular}
Consequentemente, este é o parâmetro que interessa

Tabela 1. Informações edafoclimáticas dos ambientes cultivados com os clones de Eucalyptus. 
para a predição do sucesso do melhoramento na seleção de clones, em que os valores genotípicos são preditos com base em média de várias repetições (Maia et al., 2009).

A $\mathrm{h}_{\mathrm{mg}}^{2}$ para os três caracteres avaliados nos quatro ambientes apresentou valores de alta magnitude, o que indica elevado controle genético na expressão dos caracteres (Tabela 2). Assim, há grande potencial para seleção dentro do experimento, com boas perspectivas para a seleção de clones de eucalipto de alta produtividade. Os valores de $\mathrm{h}^{2}{ }_{\mathrm{mg}}$ também evidenciaram boa precisão experimental, em razão do elevado número de repetições. Isto é de suma importância para a seleção de clones, cujos valores genéticos devem ser preditos com base nos valores fenotípicos obtidos por várias repetições. Em condições experimentais semelhantes às do presente trabalho, Garcia \& Nogueira (2005) encontraram parâmetros genéticos com magnitudes similares, o que indica que o arranjo experimental é adequado para controlar os efeitos ambientais e estimar adequadamente os valores genéticos dos caracteres avaliados.

A herdabilidade individual no sentido amplo $\left(\mathrm{h}_{\mathrm{g}}^{2}\right)-$ relativa aos efeitos genotípicos, livre da interação com os locais $\left(\mathrm{V}_{\mathrm{g}} / \mathrm{V}_{\mathrm{f}}\right)$ - apresentou valores baixos, o que reforça a importância do desdobramento da interação genótipos $\mathrm{x}$ ambientes para maximizar o ganho na seleção dos clones.

Os altos valores detectados para o coeficiente de variação genotípico $\left(\mathrm{CV}_{\mathrm{gi}}\right)$ caracterizam a existência de variabilidade genética entre os clones, para os três caracteres avaliados, e reforçam os indicativos de que grande parte da variação total é de natureza genética (Tabela 2). Segundo Resende (2002), quanto maior o valor do $\mathrm{CV}_{\mathrm{gi}}$, maiores as chances de se obterem ganhos genéticos na seleção de clones. A baixa magnitude dos valores do coeficiente de variação residual $\left(\mathrm{CV}_{\mathrm{e}}\right)$ para os caracteres DAP e ALT é coerente com o esperado para experimentos com uma planta por parcela e indica boa qualidade experimental. A adoção de uma planta por parcela, somada ao grande número de repetições, favorece o maior controle ambiental e, consequentemente, diminui o coeficiente de variação experimental (Fonseca et al., 2010). No caso do caráter VOL, espera-se que o $\mathrm{CV}_{\mathrm{e}}$ seja mais elevado, já que essa variável é obtida indiretamente, com o acúmulo dos erros experimentais das variáveis utilizadas no cálculo. Os valores de $\mathrm{CV}_{\mathrm{gi}}$ e $\mathrm{CV}_{\mathrm{e}}$ refletem a magnitude da acurácia seletiva $\left(\mathrm{AC}_{\mathrm{gen}}\right)$, que foi, em média, de 91\% para os três caracteres avaliados. A alta acurácia é indicativa de ótima relação entre os valores preditos e os reais, o que resulta em segurança na seleção de clones agronomicamente superiores (Resende \& Duarte, 2007).

Em função do maior ou do menor grau de adaptabilidade/estabilidade genética dos indivíduos, a variância da interação genótipo $\mathrm{x}$ ambiente $\left(\mathrm{V}_{\text {int }}\right)$ pode inflacionar a expressão fenotípica de um caráter (Maia et al., 2009). Essa variância se reflete diretamente no coeficiente de determinação dos efeitos da interação genótipos $\mathrm{x}$ ambientes $\left(\mathrm{C}^{2}{ }_{\text {int }}\right)$, que quantifica o quanto da variação total (fenotípica) é representada pela variação da interação genótipos x ambientes (Sturion \& Resende, 2005). As estimativas

Tabela 2. Estimativas de parâmetros genéticos (REML individual) para os caracteres diâmetro à altura do peito (DAP), altura total e volume individual total com casca, em clones de Eucalyptus, aos três anos de idade.

\begin{tabular}{lccc}
\hline Parâmetro & DAP $(\mathrm{cm})$ & Altura total $(\mathrm{m})$ & Volume individual $\left(\mathrm{m}^{3}\right)$ \\
\hline Variância genotípica & 1,162394 & 0,908291 & 0,000286 \\
Variância da interação genótipo x ambiente & 0,544272 & 0,866361 & 0,000146 \\
Variância residual & 3,174937 & 3,931596 & 0,000828 \\
Variância fenotípica individual & 4,881603 & 5,706248 & 0,001260 \\
Herdabilidade individual no sentido amplo, livre da interação & 0,238117 & 0,159175 & 0,226764 \\
Herdabilidade média & 0,869591 & 0,774827 & 0,859986 \\
Acurácia seletiva & 0,932518 & 0,880243 & 0,927354 \\
$\mathrm{R}^{2}$ da interação genótipo x ambiente & 0,111495 & 0,151827 & 0,116003 \\
Correlação genotípica do desempenho nos diversos ambientes & 0,681091 & 0,511814 & 0,661568 \\
Coeficiente de variação genotípico (\%) & 9,763320 & 5,748244 & 22,660126 \\
Coeficiente de variação residual (\%) & 16,135719 & 11,959339 & 38,577606 \\
\hline Média & 11,042801 & 16,579724 & 0,074602 \\
\hline
\end{tabular}


de $\mathrm{C}^{2}$ int para os caracteres avaliados evidenciaram que as particularidades dos ambientes em que os clones foram avaliados influenciaram a expressão fenotípica (Tabela 2). Por meio do $\mathrm{C}^{2}$ int, pode-se estimar a constância ou a previsibilidade de comportamento de um genótipo frente às variações ambientais.

$\mathrm{Na}$ análise da $\mathrm{V}_{\text {int, }}$ é possível verificar a necessidade de se discriminar esta variância no estudo da interação. A interação GxE contém uma parte relacionada à diferença entre a variância genética do caráter, nos diversos ambientes, e outra decorrente da falta de correlação entre os tratamentos genéticos de um ambiente para outro (Resende, 2007a). A primeira parcela é denominada interação simples e não acarreta maiores problemas para a seleção. Já a segunda, é denominada complexa ou cruzada e, pela falta de correlação, dificulta a seleção de genótipos de adaptação mais ampla. As magnitudes de $\mathrm{V}_{\text {int }}$ para os três caracteres, comparativamente à variância genética e à $\mathrm{h}_{\mathrm{g}}^{2}$, foi alta, o que resultou na correlação genotípica entre os ambientes (rgloc) de 68\%, para DAP, 51\%, para ALT, e 66\%, para VOL (Tabela 2). Apesar da tendência de manutenção da ordem de superioridade dos clones nos diferentes ambientes ( $\mathrm{rgloc}>50 \%$ ), os rgloc encontrados para os caracteres avaliados não são altos, o que indica a importância de se estudar sua adaptabilidade e estabilidade.

A seleção dos melhores clones foi feita pelas três diferentes estratégias (Tabelas 3, 4 e 5). Na seleção dos cinco melhores clones com base no desempenho médio em todos os ambientes (Tabela 3), com os valores genotípicos livres da interação, observou-se que o clone melhorado C6594 apresentou maior DAP e VOL. $\mathrm{O}$ ganho genético predito desse clone foi superior ao do clone comercial 386 (testemunha) (Tabela 4). Para ALT, o clone comercial foi pouco superior ao clone C6594. As magnitudes dos valores de ganho genético podem ser consideradas para recomendações de clones com vistas à seleção de material genético com desempenho médio, nos diferentes ambientes avaliados. Por causa do caráter conservador do método, que penaliza os valores genotípicos preditos, o mesmo comportamento das médias genéticas (valor genotípico predito, $\mathrm{u}+\mathrm{g}$ ) do caráter é esperado quando os clones eleitos forem submetidos a ambientes diversos (Maia et al., 2009).

Os resultados obtidos com os valores genotípicos preditos para cada ambiente $(\mathrm{u}+\mathrm{g}+\mathrm{ge})$ foram similares aos dos métodos em que se capitaliza a adaptabilidade
(PRVG) e a adaptabilidade e a estabilidade (MHPRVG) simultaneamente (Tabela 5). A escolha dos genótipos mais estáveis e mais adaptados à gama de ambientes analisados torna-se intrínseca com a capitalização da interação. Entre os métodos avaliados, esse é o que menos penaliza os valores genotípicos preditos, pois capitaliza os efeitos GxE de cada ambiente. Semelhantemente ao observado na seleção pela média genética livre de interação (Tabela 3), o clone C6594 manteve-se superior aos demais em três ambientes e esteve entre os três primeiros clones selecionados no outro ambiente (Tabela 5). No terceiro ambiente, o clone C6661 foi o que apresentou maior ganho em VOL, apesar de pouco superior ao do clone C6594. Pode-se inferir que o clone C6594 não interage de forma complexa com o ambiente, o que foi comprovado pela constância em que se apresenta na ordem de classificação nos quatro ambientes. Ao se considerar os cinco melhores genótipos selecionados para os quatro ambientes, a manutenção da ordem de superioridade dos clones é esperada para os valores encontrados de $\mathrm{V}_{\text {int }}$ e $\mathrm{C}^{2}$ int, o que caracteriza interação GxE. Os clones

Tabela 3. Estimativas de ganho genético predito para os caracteres diâmetro à altura do peito (DAP), altura total e volume individual com casca, em clones de Eucalyptus, tendo-se considerado o desempenho médio nos quatro ambientes ${ }^{(1)}$.

\begin{tabular}{|c|c|c|c|c|c|c|}
\hline Ordem & Clones & $\mathrm{g}$ & $\mathrm{u}+\mathrm{g}$ & Ganho & Nova média & $\mathrm{u}+\mathrm{g}+\mathrm{gem}$ \\
\hline & \multicolumn{6}{|c|}{ DAP } \\
\hline 1 & C6594 & 1,542 & 12,58 & 1,54 & 12,58 & 12,76 \\
\hline 2 & 386 & 1,375 & 12,41 & 1,45 & 12,50 & 12,57 \\
\hline 3 & C6515 & 1,092 & 12,12 & 1,33 & 12,37 & 12,25 \\
\hline 4 & C6661 & 0,958 & 12,00 & 1,23 & 12,28 & 12,11 \\
\hline \multirow[t]{2}{*}{5} & C6784 & 0,634 & 11,67 & 1,11 & 12,16 & 12,75 \\
\hline & \multicolumn{6}{|c|}{ Altura total } \\
\hline 1 & 386 & 1,419 & 17,99 & 1,41 & 17,99 & 18,33 \\
\hline 2 & C6594 & 1,164 & 17,74 & 1,29 & 17,87 & 18,02 \\
\hline 3 & C6661 & 0,764 & 17,34 & 1,11 & 17,69 & 17,52 \\
\hline 4 & C6572 & 0,621 & 17,20 & 0,99 & 17,52 & 17,34 \\
\hline \multirow[t]{2}{*}{5} & C6528 & 0,602 & 17,18 & 0,91 & 17,49 & 17,32 \\
\hline & \multicolumn{6}{|c|}{ Volume individual } \\
\hline 1 & C6594 & 0,026 & 0,100 & 0,026 & 0,100 & 0,104 \\
\hline 2 & 386 & 0,024 & 0,098 & 0,025 & 0,099 & 0,101 \\
\hline 3 & C6661 & 0,015 & 0,090 & 0,022 & 0,096 & 0,092 \\
\hline 4 & C6515 & 0,014 & 0,090 & 0,020 & 0,094 & 0,090 \\
\hline 5 & C6528 & 0,009 & 0,084 & 0,018 & 0,092 & 0,85 \\
\hline
\end{tabular}

${ }^{(1)} \mathrm{g}$, efeito genotípico; $\mathrm{u}+\mathrm{g}$, valor genotípico predito; $\mathrm{u}+\mathrm{g}+\mathrm{gem}$, valor genotípico médio nos ambientes. 
selecionados também podem ter sua recomendação extrapolada para plantio em vários ambientes, ao se respeitar o padrão da interação da rede experimental.

Na seleção dos cinco melhores clones para todos os ambientes (Tabela 3), verifica-se que o ganho genético predito é inferior à seleção por ambiente (Tabela 4). Isto pode ser atribuído à melhor capitalização dos efeitos da interação GxE na estratégia da seleção por ambiente, comparada à seleção para todos os ambientes baseada no valor genético.

$O$ estudo da interação GxE deve ser completado pela estimação da adaptabilidade e da estabilidade fenotípica, ou seja, o nível de resposta ao estímulo ambiental e a manutenção da produtividade frente à variação ambiental (Maia et al., 2009). Conforme mencionado, os clones que aparecem classificados em todos os ambientes como os mais produtivos não sofrem forte influência do ambiente e, consequentemente, apresentam pequena variação na interação $\mathrm{GxE}$. A seleção simultânea para produtividade, estabilidade e adaptabilidade, por meio do modelo MHPRVG (Resende, 2007a), apresenta diversas vantagens, tais como: considerar os efeitos genotípicos como aleatórios - e, dessa forma, fornecer estabilidade e adaptabilidade genotípica e não fenotípica -, fornecer valores genéticos já descontados da instabilidade e permitir computar o ganho genético com a seleção pelos três atributos, simultaneamente (Resende, 2002).

Assim como ocorreu para a seleção considerando todos os ambientes (Tabela 3), o clone C6594 apresentou melhor desempenho silvicultural com uso do modelo MHPRVG para DAP e VOL, tendo sido pouco superior ao clone comercial 386. Segundo Resende (2002), quanto menor for o desvio-padrão do comportamento genotípico através dos locais, maior será a média harmônica dos valores genotípicos através dos locais. Portanto, a seleção pelos maiores valores da média harmônica dos valores genotípicos (MHV) implica, simultaneamente, na seleção para

Tabela 4. Estimativas de ganho genético predito para os caracteres diâmetro à altura do peito (DAP), altura total e volume individual com casca, em clones de Eucalyptus, em quatro ambientes ${ }^{(1)}$.

\begin{tabular}{|c|c|c|c|c|c|c|c|c|c|c|c|c|}
\hline \multirow[t]{2}{*}{ Ambiente } & \multicolumn{4}{|c|}{ DAP } & \multicolumn{4}{|c|}{ Altura total } & \multicolumn{4}{|c|}{ Volume individual } \\
\hline & Ordem & $\mathrm{g}+\mathrm{ge}$ & $\mathrm{u}+\mathrm{g}+\mathrm{ge}$ & Ganho & Ordem & $\mathrm{g}+\mathrm{ge}$ & $\mathrm{u}+\mathrm{g}+\mathrm{ge}$ & Ganho & Ordem & $\mathrm{g}+\mathrm{ge}$ & $\mathrm{u}+\mathrm{g}+\mathrm{ge}$ & Ganho \\
\hline & \multicolumn{12}{|c|}{ Virginópolis, MG } \\
\hline \multirow{5}{*}{1} & C6594 & 1,65 & 13,57 & 1,65 & C6594 & 1,30 & 18,76 & 1,30 & C6594 & 0,028 & 0,114 & 0,028 \\
\hline & C6528 & 1,31 & 13,03 & 1,48 & C6528 & 1,23 & 18,70 & 1,27 & C6528 & 0,276 & 0,113 & 0,028 \\
\hline & 386 & 1,17 & 12,98 & 1,37 & 386 & 1,14 & 18,61 & 1,22 & 386 & 0,021 & 0,103 & 0,025 \\
\hline & C6515 & 1,13 & 12,85 & 1,31 & C6594 & 0,97 & 18,44 & 1,16 & C6515 & 0,017 & 0,107 & 0,023 \\
\hline & C6661 & 0,89 & 12,61 & 1,23 & C6502 & 0,52 & 18,03 & 1,04 & C6661 & 0,011 & 0,097 & 0,021 \\
\hline \multirow{6}{*}{2} & \multicolumn{12}{|c|}{ Santa Bárbara, MG } \\
\hline & C6594 & 1,99 & 13,43 & 1,99 & C6594 & 1,26 & 18,15 & 1,26 & C6594 & 0,033 & 0,112 & 0,033 \\
\hline & C6515 & 1,54 & 12,98 & 1,76 & 386 & 1,13 & 18,02 & 1,20 & C6515 & 0,024 & 0,104 & 0,028 \\
\hline & 386 & 1,15 & 12,59 & 1,56 & C6552 & 0,89 & 17,78 & 1,10 & 386 & 0,021 & 0,110 & 0,026 \\
\hline & C6552 & 0,70 & 12,14 & 1,34 & C6515 & 0,82 & 17,71 & 1,03 & C6661 & 0,011 & 0,091 & 0,022 \\
\hline & C6553 & 0,61 & 12,05 & 1,20 & C6661 & 0,59 & 17,48 & 0,94 & C6553 & 0,008 & 0,088 & 0,020 \\
\hline \multicolumn{13}{|c|}{ Belo Oriente, MG } \\
\hline \multirow{5}{*}{3} & C6661 & 2,28 & 12,99 & 2,28 & 386 & 2,83 & 18,36 & 2,83 & C6661 & 0,046 & 0,116 & 0,116 \\
\hline & 386 & 1,95 & 12,65 & 2,11 & C6661 & 2,70 & 18,23 & 2,77 & 386 & 0,035 & 0,104 & 0,110 \\
\hline & C6594 & 1,23 & 11,93 & 1,23 & C6776 & 1,94 & 17,47 & 2,49 & C6594 & 0,023 & 0,092 & 0,104 \\
\hline & C6553 & 1,16 & 11,87 & 1,16 & C6594 & 1,77 & 17,29 & 2,31 & C6553 & 0,016 & 0,087 & 0,100 \\
\hline & C6515 & 1,05 & 11,75 & 1,05 & C6611 & 1,34 & 16,86 & 2,12 & C6776 & 0,013 & 0,085 & 0,097 \\
\hline \multicolumn{13}{|c|}{ Cocais, MG } \\
\hline \multirow{5}{*}{4} & C6594 & 2,01 & 12,30 & 2,01 & 386 & 1,90 & 18,29 & 1,90 & C6594 & 0,033 & 0,096 & 0,033 \\
\hline & C6572 & 1,98 & 12,27 & 2,00 & C6572 & 1,89 & 18,28 & 1,90 & C6572 & 0,031 & 0,095 & 0,032 \\
\hline & 386 & 1,86 & 12,15 & 1,95 & C6594 & 1,74 & 18,13 & 1,84 & 386 & 0,031 & 0,094 & 0,032 \\
\hline & C6784 & 150 & 11,78 & 1,84 & C6528 & 1,33 & 17,72 & 1,72 & C6784 & 0,022 & 0,085 & 0,029 \\
\hline & C6552 & 1,14 & 11,43 & 1,70 & C6552 & 1,26 & 17,64 & 1,62 & C6552 & 0,017 & 0,081 & 0,027 \\
\hline
\end{tabular}

${ }^{(1)} \mathrm{g}+\mathrm{ge}$, efeito genotípico por ambiente; $\mathrm{u}+\mathrm{g}+\mathrm{ge}$, valor genotípico predito com capitalização da interação com os ambientes. 
produtividade e estabilidade. O clone C6594 também apresentou o maior valor de adaptabilidade, ou seja, apresentou o maior sinergismo adaptativo, através dos quatro ambientes analisados. Os resultados de estabilidade e adaptabilidade para o clone C6594 estão de acordo com os resultados da seleção, que anteriormente indicaram que, independentemente do ambiente, este clone tem bom desempenho (Tabelas 3 e 4). Esse mesmo padrão também foi observado para a maioria dos clones selecionados, tendo-se considerado todos os ambientes (Tabela 3), o que indica que o critério de seleção simultânea (com dados de adaptabilidade, estabilidade e produção) é eficaz para selecionar os melhores clones pelo seu valor genotípico (produção), com penalização de materiais genéticos instáveis e capitalização da adaptabilidade dos materiais mais estáveis.

Nos três métodos de seleção utilizados, fica evidente certa manutenção do ordenamento dos materiais selecionados. Isto se deve à correlação positiva e de média magnitude (Tabela 2) entre o comportamento genotípico dos clones ao longo dos diferentes ambientes. De acordo com esses resultados, podemse selecionar clones que apresentam produtividade e adaptação ampla, bem como clones que apresentam melhor desempenho em ambientes específicos.

A seleção dos cinco melhores clones pelo método MHPRVG, por meio do valor genético predito (MHPRVG*MG), resultou em uma nova média para VOL $\left(\mathrm{m}^{3}\right)$, de $0,094 \mathrm{~m}^{3}$ (Tabela 5) superior à obtida com a seleção para a produção média em todos os ambientes (média de $0,85 \mathrm{~m}^{3}$ ) (Tabela 3), porém inferior ao ganho na seleção para a produção -, em três dos quatro ambientes: médias de $0,106,0,101,0,97$ e $0,90 \mathrm{~m}^{3}$ para os ambientes $1,2,3 \mathrm{e}$ 4 , respectivamente (Tabela 4). Apesar do menor ganho na seleção, o método MHPRVG apresenta a vantagem adicional de selecionar materiais genéticos de acordo com sua adaptabilidade e estabilidade fenotípica, o que é interessante na formação de populações de melhoramento e, principalmente, na recomendação de materiais para plantios comerciais.

Tabela 5. Estabilidade e adaptabilidade (MHPRVG e MHPRVG*MG) ${ }^{(1)}$ dos valores genotípicos genéticos de clones de Eucalyptus para os caracteres diâmetro à altura do peito (DAP), altura total e volume individual.

\begin{tabular}{|c|c|c|c|c|c|c|c|c|c|}
\hline \multirow[t]{2}{*}{ Ordem } & \multicolumn{3}{|c|}{ DAP } & \multicolumn{3}{|c|}{ Altura total } & \multicolumn{3}{|c|}{ Volume individual } \\
\hline & Genótipo & MHPRVG & MHPRVG*MG & Genótipo & MHPRVG & MHPRVG*MG & Genótipo & MHPRVG & MHPRVG*MG \\
\hline 1 & C6594 & 1,1557 & 12,7625 & 386 & 1,1061 & 18,3381 & C6594 & 1,3982 & 0,1043 \\
\hline 2 & 386 & 1,1397 & 12,5855 & C6594 & 1,0875 & 18,0304 & 386 & 1,3693 & 0,1021 \\
\hline 3 & C6515 & 1,1091 & 12,2480 & C6661 & 1,0558 & 17,5042 & C6515 & 1,2122 & 0,0904 \\
\hline 4 & C6661 & 1,0939 & 12,0796 & C6528 & 1,0432 & 17,2966 & C6661 & 1,2054 & 0,0899 \\
\hline 5 & C6784 & 1,0640 & 11,7497 & C6572 & 1,0422 & 17,2797 & C6784 & 1,1388 & 0,0850 \\
\hline 6 & C6572 & 1,0564 & 11,6659 & C6776 & 1,0319 & 17,1094 & C6528 & 1,1244 & 0,0839 \\
\hline 7 & C6528 & 1,0456 & 11,5461 & C6552 & 1,0297 & 17,0729 & C6572 & 1,1148 & 0,0832 \\
\hline 8 & C6552 & 1,0341 & 11,4195 & C6515 & 1,0258 & 17,0080 & C6611 & 1,0665 & 0,0796 \\
\hline 9 & C6611 & 1,0293 & 11,3664 & C6784 & 1,0258 & 17,0068 & C6552 & 1,0487 & 0,0782 \\
\hline 10 & C6598 & 1,0216 & 11,2817 & C6611 & 1,0250 & 16,9936 & C6598 & 1,0369 & 0,0774 \\
\hline 11 & C6553 & 1,0203 & 11,2667 & C6502 & 1,0162 & 16,8489 & C6502 & 1,0355 & 0,0772 \\
\hline 12 & C6776 & 1,0188 & 11,2501 & C6553 & 1,0119 & 16,7770 & C6553 & 1,0275 & 0,0767 \\
\hline 13 & C6502 & 1,0151 & 11,2097 & C6598 & 1,0119 & 16,7770 & C6776 & 1,0247 & 0,0764 \\
\hline 14 & C6562 & 1,0068 & 11,1181 & C6562 & 0,9782 & 16,2189 & C6562 & 0,9624 & 0,0718 \\
\hline 15 & C6746 & 0,9704 & 10,7154 & C6746 & 0,9697 & 16,0779 & C6746 & 0,8689 & 0,0648 \\
\hline 16 & C6679 & 0,9204 & 10,1638 & C6752 & 0,9629 & 15,9648 & C6679 & 0,7701 & 0,0575 \\
\hline 17 & 57 & 0,9158 & 10,1131 & 57 & 0,9590 & 15,8992 & 57 & 0,7682 & 0,0573 \\
\hline 18 & C6752 & 0,8931 & 9,8629 & C6679 & 0,9297 & 15,4139 & C6679 & 0,7621 & 0,0569 \\
\hline 19 & C6677 & 0,8316 & 9,1833 & C6677 & 0,8869 & 14,7039 & C6677 & 0,5966 & 0,0445 \\
\hline 20 & C6754 & 0,8273 & 9,1362 & C6754 & 0,8822 & 14,6261 & C6754 & 0,5948 & 0,0444 \\
\hline 21 & C6735 & 0,7739 & 8,5464 & C6735 & 0,8755 & 14,5158 & C6735 & 0,5090 & 0,0380 \\
\hline
\end{tabular}

${ }^{(1)}$ MHPRVG, média harmônica do desempenho relativo dos valores genotípicos; MHPRVG*MG, refere-se à MHPRVG multiplicada pela média geral em todos os ambientes. 


\section{Conclusões}

1. A herdabilidade média dos genótipos para os três caracteres avaliados, nos quatro ambientes, apresenta valores de alta magnitude, o que indica condição favorável à seleção de clones com alta acurácia.

2. O clone C6594 apresenta o melhor desempenho nos quatro ambientes testados, de acordo com a consideração simultânea de rendimento de madeira, adaptabilidade e estabilidade.

3. O método MHPRVG proporciona seleção otimizada de clones quanto à produção, à estabilidade e à adaptabilidade e, portanto, deve fazer parte dos critérios seletivos de clones de eucalipto para construir populações de melhoramento e deve ser utilizado nas recomendações de materiais genéticos para plantios comerciais.

\section{Referências}

BASTOS, I.T.; BARBOSA, M.H.P.; RESENDE, M.D.V. de; PEDROZO, C.; MELLO, C.G.; PETERNELLI, L.A.; COSTA, P.M. de A.; XAVIER, C.V.; BAFFA, D.C.F. Correlation among predicted genotypic values and adaptability and stability estimates of sugarcane clones in a mixed models context. Scientia Agraria, v.10, p.111-118, 2009.

CRUZ, C.D.; REGAZZI, A.J.; CARNEIRO, P.C.S. Modelos biométricos aplicados ao melhoramento genético. 3.ed. Viçosa: UFV, 2004. 480p.

FONSECA, S.M. da; RESENDE, M.D.V. de; ALFENAS, A.C.; GUIMARÃES, L.M. da S.; ASSIS, T.F. de; GRATTPAGLIA, D. Manual prático de melhoramento genético do eucalipto. Viçosa: UFV, 2010. 200p.

GARCIA, C.H.; NOGUEIRA, M.C.S. Utilização da metodologia REML/BLUP na seleção de clones de eucalipto. Scientia Forestalis, v.68, p.107-112, 2005.
MAIA, M.C.C.; RESENDE, M.D.V.; PAIVA, J.R. de; CAVALCANTI, J.J.V.; BARROS, L. de M.B. Seleção simultânea para produção, adaptabilidade e estabilidade genotípicas em clones de cajueiro, via modelos mistos. Pesquisa Agropecuária Tropical, v.39, p.43-50, 2009.

PINTO JUNIOR, J.E.; STORION, J.A.; RESENDE, M.D.V.; ROZELLI JUNIOR, P. Avaliação simultânea de produtividade, adaptabilidade e estabilidade genotípica de Eucalyptus grandis em distintos ambientes do Estado de São Paulo. Boletim de Pesquisa Florestal, n.53, p.79-108, 2006.

RESENDE, M.D.V. de. Genética biométrica e estatística no melhoramento de plantas perenes. Brasília: Embrapa Informação Tecnológica; Colombo: Embrapa Florestas, 2002. 975p.

RESENDE, M.D.V. de. Matemática e estatística na análise de experimentos e no melhoramento genético. Colombo: Embrapa Florestas, 2007a. 362p.

RESENDE, M.D.V. de. SELEGEN-REML/BLUP: sistema estatístico e seleção genética computadorizada via modelos lineares mistos. Colombo: Embrapa Florestas, 2007b. 359p.

RESENDE, M.D.V. de; DUARTE, J.B. Precisão e controle de qualidade em experimentos de avaliação de cultivares. Pesquisa Agropecuária Tropical, v.37, p.182-194, 2007.

STURION, J.A.; RESENDE, M.D.V. de. Seleção de progênies de erva-mate (Ilex paraguariensis St. Hil.) para produtividade, estabilidade e adaptabilidade temporal de massa foliar. Boletim de Pesquisa Florestal, n.50, p.37-51, 2005.

SUDARIC, A.; SIMIC, D.; VRATARIC, M. Characterization of genotype by environment interactions in soybean breeding programmes of southeast Europe. Plant Breeding, v.125 p.191-194, 2005.

VERARDI, C.K.; RESENDE, M.D.V. de; COSTA, R.B. da; GONÇALVES, P. de S. Adaptabilidade e estabilidade da produção de borracha e seleção em progênies de seringueira. Pesquisa Agropecuária Brasileira, v.44, p.1277-1282, 2009.

ZENI-NETO, H.; OLIVEIRA, R.A. de; DAROS, E.; BESPALHOK-FILHO, J.C.; ZAMBON, J.L.C.; WEBER, H. Seleção para produtividade, estabilidade e adaptabilidade de clones de cana-de-açúcar em três ambientes no Estado do Paraná via modelos mistos. Scientia Agraria, v.9, p.425-430, 2008.

Recebido em 25 de janeiro de 2012 e aprovado em 5 de junho de 2012 